
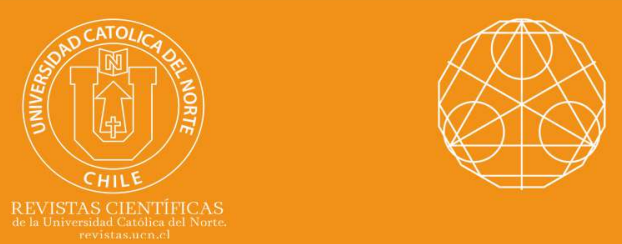

\title{
A general common fixed point result for two pairs of maps in b-metric spaces
}

Mohamed Akkouchi ${ }^{1}$

${ }^{1}$ University Cadi Ayyad, Dept. of Mathematics, Faculty of Sciences-Semlalia, Marrakesh, Morocco.

akkm555@yahoo.fr

\section{Abstract:}

We establish a general common fixed point problem for two pairs $\{f, S\}$ and $\{g, T\}$ of weakly compatibles selfmaps of a complete b-metric $(X$, $d ; s)$. These maps are satisfying a contractive condition defined by $a$ class of implicit relations in five variables. This contraction unifies, in one go, several contractive conditions previously used in a set of recent papers dealing with fixed point or common fixed results for selfmaps of b-metric spaces. We provide an illustrative example.

Keywords: Complete b-metric spaces; Implicit relations; Common fixed point for four maps; Weakly compatible maps; Compatible maps.

MSC (2020): 54H25, 47H10

\section{Cite this article as (IEEE citation style):}

M. Akkouchi, "A general common fixed point result for two pairs of maps in b-metric spaces", Proyecciones (Antofagasta, On line), vol. 39 , no. 5, pp. 1251-1266, Oct. 2020, doi: 10.22199/issn.0717-6279-2020-05-0076

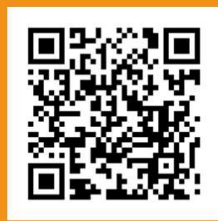

Article copyright: (C) 2020 Mohamed Akkouchi. This is an open access article distributed under the terms of the Creative Commons Licence, which permits unrestricted use and distribution provided the original author and source are credited. 


\section{Introduction and preliminaries}

Throughout this paper, $(X, d ; s)$ will be a $b$-metric space with constant $s \geq 1$.

We recall the following definition of b-metric spaces (see [5], [8], [6], [9]; [18], [15], [7]).

Definition 1.1. Let $X$ be a nonempty set. $A$ b-metric on $X$ is a function $d: X \times X \rightarrow[0, \infty)$ satisfying the conditions

(i) $\quad d(x, y)=0 \Longleftrightarrow x=y$

(ii) $\quad d(x, y)=d(y, x)$,

(iii) $\quad d(x, y) \leq s[d(x, z)+d(z, y)]$,

for all $x, y, z \in X$, and for some fixed number $s \geq 1$.

The triple $(X, d ; s)$ is called a b-metric space with parameter $s$.

The inequality (iii) is called the s-relaxed triangle inequality or simply the s-triangle inequality.

It is clear that when $s=1$, the function $d$ becomes a metric on $X$. In this case the triple $(X, d ; 1)$ is simply denoted by $(X, d)$ which is the usual notation for a metric space $X$ endowed with the metric $d$.

Let $(X, d ; s)$ be a $b$-metric space with constant $s \geq 1$. The ball $B(x, r)$ of center $x \in X$ and radius $r>0$ is defined by setting

$\mathrm{B}(\mathrm{x}, \mathrm{r})=\{\mathrm{y} \in X: d(x, y)<r\}$. A nonempty subset $Y$ of $X$ is called open if for every $x \in Y$ there exists a number $r_{x}>0$ such that $B\left(x, r_{x}\right) \subset Y$. The empty set is open by definition.

We denote by $\mathcal{T}_{d}$ the family of all open subsets of $X$ it easy to see that $\mathcal{T}_{d}$ satisfies the axioms of a topology. It is known that this topology $\mathcal{T}_{d}$ is metrizable (see for example [7] and the references therein). As a consequence, for every sequence $\left(x_{n}\right)$ and for each $x \in X$, we have the following equivalence: $\left(x_{n}\right)$ converges to $x$ in the topological space $\left(X, \mathcal{T}_{d}\right)$ if, and only if, $\lim _{n \rightarrow+\infty} d\left(x_{n}, x\right)=0$.

If $\lim _{n \rightarrow+\infty} d\left(x_{n}, x\right)=0$, then we write $\lim _{n \rightarrow+\infty} x_{n}=x$.

It is easy to see that the topological space $\left(X, \mathcal{T}_{d}\right)$ is Hausdorff. This implies that the limit of a converging sequence is unique. 
Contrary to the usual metric spaces, the map $(x, y) \rightarrow d(x, y)$ fails to be continuous on the topological product space $X \times X$ and the balls $B(x, r)$ are not be open sets (see for instance [7] and [18]).

A sequence $\left(x_{n}\right)_{n \geq 0}$ of points of $X$ is said to be Cauchy sequence if, and only if $\lim _{n, m \rightarrow+\infty} d\left(x_{n}, x_{m}\right)=0$.

It is easy to see that every converging sequence is a Cauchy sequence.

The following fundamental lemma was improved in 2017 by R. Miculescu and A. Mihail in their paper [16] and by T. Suzuki in his paper [20] by a different method.

Lemma 1.1 ([16] and [20]). Let $(X, d ; s)$ be a b-metric space with parameter $s \geq 1$. Let $\left(x_{n}\right)_{n \in N}$ be a sequence of elements from $X$ having the property that there exists $\gamma \in[0,1)$ such that

$d\left(\mathrm{x}_{n+1}, x_{n}\right) \leq \gamma d\left(x_{n}, x_{n-1}\right), \quad \forall n \in N$.

Then the sequence $\left(x_{n}\right)_{n \in N}$ is Cauchy.

In [10], T. M. Došenović, M. V. Pavlović and S. N. Radenović made several important observations concerning Lemma 1.1 above and showed how various known fixed point results in b-metric spaces can be shortened by using this lemma.

The b-metric space $(X, d, s)$ is said to be complete iff every Cauchy sequences converges in $X$.

A subset $Y$ of $X$ is said to be closed if the set $Y^{c}:=X \backslash Y$ is open (i.e., $\left.Y^{c} \in \mathcal{T}_{d}\right)$.

Let $Y$ be a non empty set of the b-metric space $(X, d ; s)$. We denote $d_{Y}$ the restriction of d to the set $Y \times Y$. Then the space $\left(Y, d_{Y} ; s\right)$ is b-metric space, called a b-metric subspace of $X$. We observe that if $\left(Y, d_{Y} ; s\right)$ is a complete b-metric space, then $Y$ is closed in $(X, d ; s)$.

For each subset $Y$ of $X$, we denote $\bar{Y}$ the closure of $Y$. That is the smallest closed subset of $X$ containing $Y$.

It is easy to prove the following two lemma.

Lemma 1.2. Let $(X, d ; s)$ be a b-metric space and let $Y$ be a subset of $X$. Let $x \in X$. Then the following assertions are equivalent:

(a) $x \in \bar{Y}$.

(b) There exists a sequence $\left(y_{n}\right)_{n}$ of points in $Y$ which converges to $x$.

(c) $d(x, Y)=0$, where as usual, $d(x, Y):=\inf \{d(x, y): y \in Y\}$.

The following lemma may be obtained as a direct consequence from Lemma 1.2 . 
Lemma 1.3. Let $(X, d ; s)$ be a b-metric space and let $Y$ be a subset of $X$. Then the following assertions are equivalent:

(a) $Y$ is closed.

(b) $Y=\bar{Y}$.

(c) For each sequence $\left(y_{n}\right)_{n}$ of points in $Y$ and for every $x \in X$, if $\lim _{n \rightarrow+\infty} d\left(x, y_{n}\right)=0$, then we have $x \in Y$.

From the lemma above, we deduce that every closed subset of a complete bmetric space is complete (i.e., the subspace $\left(Y, d_{Y} ; s\right)$ is a complete b-metric space).

The following definition was introduced by Jungck in 1996 (see [14]) in the setting of metric fixed point theory.

Definition 1.2. ([14]) Two selfmaps $f$ and $g$ of a nonempty set $X$ are called weakly compatible maps if they commute at every coincidence point of $f$ and $g$ (i.e., for all $u \in X, f u=g u \Longrightarrow f g(u)=g f(u)$ ).

The following definition extends the concept of weak compatibility introduced by Jungck in 1986 (see [13]) for selfmaps of metric spaces.

Definition 1.3. Two self-mappings $S$ and $T$ of a $b$-metric space $(X, d ; s)$ are called b-compatible if $\lim _{n \rightarrow \infty} d\left(S T x_{n}, T S x_{n}\right)=0$, whenever $\left\{x_{n}\right\}$ is a sequence in $X$ such that $\lim _{n \rightarrow \infty} S x_{n}=\lim _{n \rightarrow \infty} T x_{n}=t$, for some $t$ in $X$.

We point out that another kind of compatibility for maps was recently used in [4] to investigate common fixed point theorems for two pairs of selfmaps of b-metric spaces satisfying a Lipshitz contractive condition.

As before, let $(X, d ; s)$ be a $b$-metric space with constant $s \geq 1$. Let $I, J, S$ and $T$ be selfmaps of $X$ satisfying the following general contractive conditions:

$$
d(S x, T y) \leq f\left(d(I x, J y), d(I x, S x), d(J y, T y), \frac{d(I x, T y)}{s}, \frac{d(J y, S x)}{s}\right)
$$

holds for all $x, y \in X$, where $f:\left([0, \infty)^{5} \rightarrow[0, \infty)\right.$ satisfying certain conditions (see Section 2).

The aim of this paper is to establish two general common fixed point theorems (see Theorem 3.1 and Theorem 3.2) for these self-mappings $I, J, S$ and $T$, when the pairs $(S, I)$ and $(T, J)$ are weakly compatible. We point out that our results do not make appeal to continuity. 
As a consequence of our general results, we recapture several earlier fixed point results dealing with some contractive conditions that can be entered as particular cases. So our results allow us to unify several related results existing in recent literature.

We point out that this paper extend certain results investigated in [1] (for metric case) to the more general context of b-metric spaces. We point out also, that the author has used implicit relations in [2] and [3] to study fixed point results in b-metric spaces.

This paper is organized as follow:

In Section 2, after this introduction and preliminaries, we introduce the class of functions used to define the contractive condition (1.2). We provide also examples of such implicit relations.

In Section 3, we establish our main results (see Theorem 3.1 and Theorem 3.2).

In Section 4, we provide several consequences, where some previousely published fixed point results are seen as particular cases of our general results.

\section{Implicit relations}

For all number $s \geq 1$, we consider the set $\mathcal{F}_{s, 5}$ of all real functions $f$ : $\left([0, \infty)^{5} \mapsto[0, \infty)\right.$ satisfying the following conditions:

$\left(F_{1}\right)$ : is upper semi-continuous on $[0, \infty)^{5}$ and nondecreasing in variables $x_{4}$ and $x_{5}$;

$\left(F_{2}\right): f(0,0, u, u, 0)<\frac{u}{s}$, for all $u>0$;

$\left(F_{3}\right): f\left(u, 0,0, \frac{u}{s}, \frac{u}{s}\right)<u$, for all $u>0$;

$\left(F_{4}\right)$ : there exist $0 \leq h<1$ such that for every $u, v \in[0, \infty)$ with

$\left(F_{4, a}\right): u \leq f(v, v, u, u+v, 0)$, or

$\left(F_{4, b}\right): u \leq f(v, u, v, 0, u+v)$

we have $u \leq h v$.

Next we give some examples of such functions.

Example 2.1. $f\left(t_{1}, \ldots, t_{5}\right)=\lambda \max \left\{t_{1}, t_{2}, t_{3}, \frac{1}{2}\left(t_{4}+t_{5}\right)\right\}$, where $\lambda \in\left[0, \frac{1}{s}\right)$.

$\left(F_{1}\right)$ : is clear.

$\left(F_{2}\right): f(0,0, u, u, 0)=\lambda u=s \lambda \cdot \frac{u}{s}<\frac{u}{s}$, for all $u>0$.

$\left(F_{3}\right): f\left(u, 0,0, \frac{u}{s}, \frac{u}{s}\right)=\lambda u<u$ for all $u>0$.

$\left(F_{4}\right)$ : For all $u, v \geq 0$, we have

$$
f(v, v, u, u+v, 0)=\lambda \max \left\{u, v, \frac{1}{2}(u+v)\right\}=\lambda \max \{u, v\} .
$$


Suppose that $u \leq f(v, v, u, u+v, 0)$ (or $u \leq f(v, u, v, 0, u+v)$ ) and that $u>v$. Then, from (2.1.a), we get $u(1-\lambda) \leq 0$, a contradiction. Therefore $u \leq v$, which yields (by (2.1)) that $u \leq \lambda v$. Thus $F_{4, a}$ is true with $h:=\lambda \in$ $\left[0, \frac{1}{s}\right)$. For all $u, v \geq 0$, we have

$$
f(v, u, v, 0, u+v)=\lambda \max \left\{u, v, \frac{1}{2}(u+v)\right\}=\lambda \max \{u, v\} .
$$

Suppose that $u \leq f(v, u, v, 0, u+v)$ and that $u>v$. Then, from (2.1.b), we get $u(1-\lambda) \leq 0$, a contradiction. Therefore $u \leq v$, which yields (by (2.1.b)) that $u \leq \lambda v$. Thus $F_{4, b}$ is true with the sdame $h:=\lambda \in\left[0, \frac{1}{s}\right)$.

Example 2.2. $f\left(t_{1}, \ldots, t_{5}\right)=a_{1} t_{1}+a_{2} t_{2}+a_{3} t_{3}+a_{4} t_{4}+a_{5} t_{5}$, where $a_{i} \geq 0$ for $i=1,2, \ldots, 5$; with $a_{3}+a_{4}<\frac{1}{s}$ and $a_{1}+a_{2}+a_{3}+2 \max \left\{a_{4}, a_{5}\right\}<1$.

$\left(F_{1}\right)$ : is clear.

$\left(F_{2}\right): f(0,0, u, u, 0)=\left(a_{3}+a_{4}\right) u=\left(s\left(a_{3}+a_{4}\right)\right) \frac{u}{s}<\frac{u}{s}$, for all $u>0$.

$\left(F_{3}\right): f\left(t, 0,0, \frac{u}{s}, \frac{u}{s}\right)=u\left(a_{1}+\frac{a_{4}+a_{5}}{s}\right)<u$, for all $u>0$. (Because $\left.a_{1}+\frac{a_{4}+a_{5}}{s}<1\right)$.

$\left(F_{4}\right)$ : For all $u, v \geq 0$, we have

$$
f(v, v, u, u+v, 0)=\left(a_{3}+a_{4}\right) u+\left(a_{1}+a_{2}+a_{4}\right) v .
$$

If $u \leq f(v, v, u, u+v, 0)$, then $u \leq h_{1} v$, where $h_{1}:=\frac{a_{1}+a_{2}+a_{4}}{1-a_{3}-a_{4}}$.

Also, we have

$$
f(v, u, v, 0, u+v)=\left(a_{2}+a_{5}\right) u+\left(a_{1}+a_{3}+a_{5}\right) v .
$$

If $u \leq f(v, u, v, 0, u+v)$, then $u \leq h_{2} v$, where $h_{2}:=\frac{a_{1}+a_{3}+a_{5}}{1-a_{2}-a_{5}}$.

We set $h:=\max \left\{h_{1}, h_{2}\right\}$. Then by assumtion, we have $h \in[0,1)$ and from $(2.2)$ and $(2.3),\left(F_{4}\right)$ is satisfied with the prameter $h$.

Example 2.3. $f\left(t_{1}, \ldots, t_{5}\right)=\lambda t_{1}$, where $\lambda \in(0,1)$.

This is a particular case of Example 2.2, where $a_{1}=\lambda$ and $a_{2}=a_{3}=$ $a_{4}=a_{5}=0$.

Example 2.4. $f\left(t_{1}, \ldots, t_{5}\right)=\left(a t_{1} t_{2}+b t_{2} t_{3}+c t_{4} t_{5}\right)^{\frac{1}{2}}$, where $a>0 ; b, c \geq$ $0 ; a+b<1$ and $c<s^{2}$.

$\left(F_{1}\right)$ : is evident.

$\left(F_{2}\right): f(0,0, u, u, 0)=0<\frac{u}{s}$, for all $u>0$.

$\left(F_{3}\right): f\left(u, 0,0, \frac{u}{s}, \frac{u}{s}\right)=\left[c \frac{u^{2}}{s u^{2}}\right]^{\frac{1}{2}}=\frac{\sqrt{c}}{s} u<u$, because $\sqrt{c}<s$.

$\left(F_{4}\right)$ : For all $u, v \geq 0$, we have

$$
u \leq f(v, v, u, u+v, 0) \Rightarrow u^{2}-a v^{2}-b u v \leq 0 .
$$


Let $v>0$ and $g(t)=t^{2}-b t-a$, where $t=\frac{u}{v}$. We observe that $g(0)=-a<0$ and $g(1)=1-(a+b)>0$. Then there exists $r \in(0,1)$ suc that $g(r)=0$. In fact we have $r=\frac{b+\sqrt{b^{2}+4 a}}{2}$. Since the other root of the equation $g(t)=0$ is strictly negative, then the inequality $g(t) \leq 0(t \geq 0)$ implies that $t \leq r$. Thus, if $u \leq f(v, v, u, u+v, 0)$ with $v>0$, then we have $u \leq r v$. If $v=0$, then from (2.3) we get $u=0$. Therefore $u \leq r v$.

Also, for all $u, v \geq 0$, we have

$$
u \leq f(v, u, v, 0, u+v) \Rightarrow u^{2} \leq a u v+b u v \Rightarrow u \leq(a+b) v .
$$

By setting $h:=\max \left\{a+b, \frac{b+\sqrt{b^{2}+4 a}}{2}\right\}$, we see that $h \in[0,1)$ and that the condition $\left(F_{4}\right)$ is fulfilled with this constant.

\section{Main results}

As before, $X$ denotes a b-metric space endowed with the b-metric $d$ with parameter $s \geq 1$. We recall that for any subset $A$ of $X$, the closure of $A$ is denoted by $\bar{A}$.

Our first main result reads as follows.

Theorem 3.1. Let $I, J, S$ and $T$ be mappings from a complete b-metric space $(X, d ; s)$ into itself satisfying the conditions:

(A 1) $S(X) \subset J(X)$ and $T(X) \subset I(X)$.

(A 2) The pairs $(S, I)$ and $(T, J)$ are weakly compatible.

(A 3) $\overline{S(X)} \cap \overline{T(X)} \subset I(X) \cup J(X)$.

(A 4) The inequality

$(3.1) d(S x, T y) \leq f\left(d(I x, J y), d(I x, S x), d(J y, T y), \frac{d(I x, T y)}{s}, \frac{d(J y, S x)}{s}\right)$,

holds for all $x, y \in X$, where $f \in \mathcal{F}_{s, 5}$.

Then $I, J, S$ and $T$ have a unique common fixed point $z$.

Furtheremore, we have $\{z\}=F i x\{S, I\}=F i x\{T, J\}$.

Proof. Let $x_{0}$ be an arbitrary point in $X$. Set $y_{0}=S x_{0}$. Since $S(X) \subset$ $J(X)$, then we can find a point $x_{1} \in X$ such that $y_{0}=J x_{1}$. Set $y_{1}=T x_{1}$. Since $T(X) \subset I(X)$, then we can find a point $x_{2} \in X$ such that $y_{1}=I x_{2}$. By induction it is easy to construct two sequences $\left(x_{n}\right)$ and $\left(y_{n}\right)$ in $X$ satisfying for each nonnegative integer $n$, 


$$
y_{2 n}=S x_{2 n}=J x_{2 n+1} \quad \text { and } \quad y_{2 n+1}=T x_{2 n+1}=I x_{2 n+2}
$$

For each non negative integer $n$, we set $d_{n}:=d\left(y_{n}, y_{n+1}\right)$. Using inequality (3.1), the property $\left(F_{1}\right)$ and the $s$-triangle inequality, for all nonnegative integer $n$, we have :

$$
\begin{aligned}
d_{2 n+1} & =d\left(y_{2 n+2}, y_{2 n+1}\right)=d\left(S x_{2 n+2}, T x_{2 n+1}\right) \\
& \leq f\left(d\left(I x_{2 n+2}, J x_{2 n+1}\right), d\left(I x_{2 n+2}, S x_{2 n+2}\right), d\left(J x_{2 n+1}, T x_{2 n+1}\right),\right. \\
& \left.\frac{1}{s} d\left(I x_{2 n+2}, T x_{2 n+1}\right), \frac{1}{s} d\left(J x_{2 n+1}, S x_{2 n+2}\right)\right) \\
& =f\left(d\left(y_{2 n+1}, y_{2 n}\right), d\left(y_{2 n+1}, y_{2 n+2}\right), d\left(y_{2 n}, y_{2 n+1}\right), 0, \frac{1}{s} d\left(y_{2 n}, y_{2 n+2}\right)\right) \\
& =f\left(d_{2 n}, d_{2 n+1}, d_{2 n}, 0, \frac{d\left(y_{2 n}, y_{2 n+2}\right)}{s}\right) \\
& \leq f\left(d_{2 n}, d_{2 n+1}, d_{2 n}, 0, d_{2 n}+d_{2 n+1}\right) .
\end{aligned}
$$

By $\left(F_{3, b}\right)$, we obtain $d_{2 n+1} \leq h d_{2 n}$. Similarly, by $\left(F_{3, a}\right)$ we obtain $d_{2 n} \leq h d_{2 n-1}$ for all positive integer $n$. We deduce that

$$
d_{n} \leq h^{n} d_{0},
$$

for all non negative integers $n$.

By Lemma 1.1, it follows that the sequence (3.2) is a Cauchy sequence. Since $X$ is complete, the sequence $\left\{y_{n}\right\}$ converges to some element $z \in X$. Thus, we have

$$
z=\lim _{n \rightarrow \infty} S x_{2 n}=\lim _{n \rightarrow \infty} J x_{2 n+1}=\lim _{n \rightarrow \infty} T x_{2 n+1}=\lim _{n \rightarrow \infty} I x_{2 n+2} .
$$

By assumption (A 3), we have $z \in I(X) \cup J(X)$.

(1) Suppose that $z$ belongs to the set $J(X)$. Then we can find a point $u \in X$ so that $z=J u$. We set $\tau:=d(T u, z)$. To get a contradiction, let us suppose that $\tau>0$. Then by using the inequality (3.1), the $s$-triangle inequality and the property $\left(F_{1}\right)$ of $f$,we have successively

$$
\begin{aligned}
d(T u, z) & \leq s d\left(T u, S x_{2 n}\right)+s d\left(S x_{2 n}, z\right) \\
& \leq s f\left(d\left(I x_{2 n}, z\right), d\left(I x_{2 n}, S x_{2 n}\right), d(z, T u), \frac{1}{s} d\left(I x_{2 n}, T u\right)\right. \\
& \frac{1}{s} d\left(z, S x_{2 n}\right)+s d\left(S x_{2 n}, z\right) \\
& \leq s f\left(d\left(I x_{2 n}, z\right), d\left(I x_{2 n}, S x_{2 n}\right), d(z, T u), d\left(I x_{2 n}, z\right)+d(z, T u),\right. \\
& \left.d\left(z, S x_{2 n}\right)\right)+s d\left(S x_{2 n}, z\right) .
\end{aligned}
$$

Again, by the $s$-triangle inequality, for all nonnegative integer $n$, we have

$d\left(I x_{2 n}, S x_{2 n}\right) \leq s d\left(I x_{2 n}, z\right)+s d\left(z, S x_{2 n}\right)$ (which tend to zero when $\left.n \longrightarrow+\infty\right)$. 
By taking the limits as $n \mapsto \infty$ in both sides of the above inequalities, we get

$$
0<\tau \leq s f(0,0, \tau, \tau, 0)
$$

which is a contradiction with $\left(F_{2}\right)$. Thus we have $z=J u=T u$.

Since $T(X) \subset I(X)$, there exists a point $v \in X$ such that $z=I v$. Then, again by using the inequality (3.1), we get

$d(S v, z)=d(S v, T u) \leq f\left(0, d(z, S v), 0,0, \frac{1}{s} d(S v, z)\right) \leq f(0, d(z, S v), 0,0, d(S v, z))$.

By the property $\left(F_{4, b}\right)$ it follows that $S v=z$. So we have proved that

$$
J u=T u=z=S v=I v .
$$

Since the pairs $(T, J)$ and $(S, I)$ are weakly compatible then we have

$$
T J u=J T u, \quad \text { and } \quad S I v=I S v .
$$

From (3.3) we obtain

$$
T z=J z \quad \text { and } \quad S z=I z .
$$

Now, we show that $z$ is a fixed point of $S$. We set $\tau:=d(S z, z)$ and suppose that $\tau>0$. Then by using the inequalities (3.1) and (3.4), we have

$$
\begin{aligned}
0<\tau & =d(S z, T u) \\
& \leq f\left(d(I z, J u), d(I z, S z), d(J u, T u), \frac{1}{s} d(I z, T u), \frac{1}{s} d(S z, J u)\right) \\
& =f\left(\tau, 0,0, \frac{\tau}{s}, \frac{\tau}{s}\right),
\end{aligned}
$$

which is in contradiction with the property $\left(F_{3}\right)$. Thus $S z=z$. Therefore $S z=I z=z$.

Set $\rho:=d(z, T z)$ and suppose that $\rho>0$. Then by using the condition (3.1) and the inequality (3.4), we have

$$
\begin{aligned}
0<\rho & =d(z, T z)=d(S z, T z) \\
& \leq f\left(d(I z, J z), d(I z, S z), d(J z, T z), \frac{1}{s} d(I z, T z), \frac{1}{s} d(J z, S z)\right) \\
& \left.=f\left(d(z, T z), d(z, z), d(T z, T z), \frac{1}{s} d(z, T z), \frac{1}{s} d(T z, z)\right)\right) \\
& =f\left(\rho, 0,0, \frac{\rho}{s}, \frac{\rho}{s}\right)
\end{aligned}
$$

a contradiction with $\left(F_{3}\right)$. Thus $J z=T z=z$. Therefore $z$ is a common fixed point of the mappings $I, J, S$ and $T$.

(2) Suppose that $z$ belongs to the set $I(X)$. Then by similar arguments, one can prove as it is done in (1) that $z$ is a common fixed point of the mappings $I, J, S$ and $T$. 
(3) The uniqueness of $z$ is a consequence of conditions (3.1) and the property $\left(F_{2}\right)$.

(4) Now we show that $z$ is the unique common fixed point of the mappings $S$ and $I$. Suppose that there exists $w \in F i x\{S, I\}$ with $w \neq z$. Then by using the condition (3.1), we have

$$
\begin{aligned}
0<d(z, w) & =d(S w, T z) \\
& \leq f\left(d(I w, J z), d(I w, S w), d(J z, T z), \frac{1}{s} d(I w, T z), \frac{1}{s} d(J z, S w)\right) \\
& =f\left(d(w, z), 0,0, \frac{d(w, z)}{s}, \frac{d(w, z)}{s}\right),
\end{aligned}
$$

contradicting $\left(F_{2}\right)$. Therefore $\{z\}=F i x\{S, I\}$.

Suppose that there exists $v \in F i x\{T, J\}$ with $v \neq z$. Then by using the condition (3.1), we have

$$
\begin{aligned}
0<d(z, v) & =d(S z, T v) \\
& \leq f\left(d(I z, J v), d(I z, S z), d(J v, T v), \frac{1}{s} d(I z, T v), \frac{1}{s} d(J v, S z)\right) \\
& =f\left(d(z, v), 0,0, \frac{d(z, v)}{s}, \frac{d(v, z)}{s}\right),
\end{aligned}
$$

contradicting $\left(F_{3}\right)$. Therefore $\{z\}=F i x\{T, J\}$. This ends the proof.

Remark. Let $I, J, S$ and $T$ be mappings from a b-metric space $(X, d ; s)$ into itself satisfying the conditions:

(A 1$) S(X) \subset J(X)$ and $T(X) \subset I(X)$ and

(A 3)' One of the sets $S(X), T(X), I(X)$ or $J(X)$ is complete.

Then it is easy to see that $I, J, S$ and $T$ are also satisfying the condition (A 3).

Therefore, by a proof similar to the one given for Theorem 3.1, one can obtain the following result in (possibly non complete) b-metric spaces.

Theorem 3.2. Let $I, J, S$ and $T$ be mappings from a $b$-metric space $(X, d ; s)$ into itself satisfying the conditions:

(A 1) $S(X) \subset J(X)$ and $T(X) \subset I(X)$.

(A 2) The pairs $(S, I)$ and $(T, J)$ are weakly compatible.

(A 3)' One of the sets $S(X), T(X), I(X)$ or $J(X)$ is complete.

(A 4) The inequality

$(3.3) d(S x, T y) \leq f\left(d(I x, J y), d(I x, S x), d(J y, T y), \frac{d(I x, T y)}{s}, \frac{d(J y, S x)}{s}\right)$,

holds for all $x, y \in X$, where $f \in \mathcal{F}_{s, 5}$.

Then $I, J, S$ and $T$ have a unique common fixed point $z$.

Furtheremore, we have $\{z\}=F i x\{S, I\}=F i x\{T, J\}$. 
We end this section by giving an illustrative example.

\section{Example 3.1.}

Let $X:=[0,1]$ be equipped with the b-metric $d$ given by $d(x, y):=$ $|x-y|^{2}$ for all $x, y \in X$. The parameter of the b-metric $d$ is $s=2$. We define four maps $I, J, S$ and $T$ on $X$ by setting

$$
S(x):=\frac{x^{8}}{81}, \quad T(x):=\frac{x^{4}}{16}, \quad I(x):=\frac{x^{4}}{9} \quad \text { and } \quad J(x):=\frac{x^{2}}{4} .
$$

Then we have:

(1) The b-metric space $(X, d ; 2)$ is complete.

(2) The inclusions $S X \subseteq J X$ and $T X \subseteq I X$ are evident.

(3) The inclusion $\overline{S(X)} \cap \overline{T(X)} \subset I(X) \cup J(X)$ is obvious.

(4) It is easy to see that the pairs $\{S, I\}$ and $\{T, J\}$ are weakly compatible.

(5) For all $x, y \in X$, we have

$$
\begin{aligned}
d(S x, T y) & =|S x-T y|^{2}=\left(\frac{x^{4}}{9}+\frac{y^{2}}{4}\right)^{2}\left(\frac{x^{4}}{9}-\frac{y^{2}}{4}\right)^{2} \\
& \leq\left(\frac{x^{4}}{9}+\frac{y^{2}}{4}\right)^{2} d(I x, J y) \\
& \leq \frac{13^{2}}{36^{2}} d(I x, J y) \leq \frac{5}{24} d(I x, J y) \\
& \leq \frac{5}{24} \max \left\{d(I x, J y), d(S x, I x), d(T y, J y), \frac{d(S x, J y)+d(T y, I x)}{4}\right\} .
\end{aligned}
$$

We set $\lambda=\frac{5}{24}$. We observe, here, that $s \lambda=2 \lambda=\frac{5}{12}<1$.

According to the item (5) above, we see that the assumption (A 4) is satisfied with the function $f$ given by:

$$
f\left(t_{1}, \ldots, t_{5}\right):=\frac{5}{24} \max \left\{t_{1}, t_{2}, t_{3}, \frac{1}{2}\left(t_{4}+t_{5}\right)\right\}, \text { for all }\left(t_{1}, t_{2}, t_{3}, t_{4}, t_{5}\right) \in
$$
$[0, \infty)^{5}$.

We conclude that all conditions of Theorem 3.1 are satisfied and the unique common fixed point of the selfmappings $S, T, I$ and $J$ is the point zero.

\section{Some consequences and related results}

A first consequence One of the first consequences of Theorem 3.2 is the following theorem.

Theorem 4.1. Let $I, J, S$ and $T$ be mappings from a $b$-metric space $(X, d ; s)$ into itself satisfying the conditions:

(A 1) $S(X) \subset J(X)$ and $T(X) \subset I(X)$.

(A 2) The pairs $(S, I)$ and $(T, J)$ are weakly compatible. 
(A 3)' One of the sets $S(X), T(X), I(X)$ or $J(X)$ is complete.

(A 4)' $d(S x, T y) \leq \lambda d(I x, J y)$ holds for all $x, y \in X$, where $\lambda$ is a constant in $[0,1)$.

Then $I, J, S$ and $T$ have a unique common fixed point $z$.

Furtheremore, we have $\{z\}=F i x\{S, I\}=F i x\{T, J\}$.

We point out that the condition $0 \leq \lambda<{ }_{s}^{1}$ imposed in [12] is relaxed here in Theorem 4.1. Thus our second main result gives an ameloration to [Theorem 3.13 in [12]].

We give next a result which is related to the first main result.

Theorem 4.2. Let $f \in \mathcal{F}_{s, 5}$ and let $I, J, S$ and $T$ be mappings from a complete $b$-metric space $(X, d ; s)$ into itself satisfying for some positive integers $p, q, r$ and $s$,

$\left.d\left(S^{p} x, T^{q} y\right) \leq f\left(d\left(I^{r} x, J^{s} y\right)\right), d\left(I^{r} x, S^{p} x\right), d\left(J^{s} y, T^{q} y\right), \frac{1}{s} d\left(I^{r} x, T^{q} y\right), \frac{1}{s} d\left(J^{s} y, S^{p} x\right)\right)$

for all $x \in X$.

Suppose that

(A 1) $S^{p}(M) \subset J^{s}(M)$ and $T^{q}(M) \subset I^{r}(M)$.

(A 2) The pairs $(S, I)$ and $(T, J)$ are commuting.

(A 3) $\overline{S^{p}(M)} \cap \overline{T^{q}(M)} \subset I^{r}(M) \cup J^{s}(M)$.

Then $I, J, S$ and $T$ have a unique common fixed point $z$. Furtheremore, we have $\{z\}=F i x\{S, I\}=F i x\{T, J\}$.

Proof. Set $\tilde{S}:=S^{p}, \tilde{T}:=T^{q}, \tilde{I}:=I^{r}$ and $\tilde{J}:=J^{s}$. Then an application of Theorem 2.1 yields that the mappings $\tilde{I}, \tilde{J}, \tilde{S}$ and $\tilde{T}$ have a unique common fixed point $z$ in $M$. We have $S z=\tilde{S}(S z)$ and by the commutativity of $S$ and $I$ we have $S z=S\left(I^{r} z\right)=I^{r}(S z)=\tilde{I}(S z)$. Thus $S z \in F i x\{\tilde{S}, \tilde{I}\}$. By virtue of Theorem 2.1, we must have $S z=z$. By similar arguments we have $I z=z$ and $J z=T z=z$.

The following theorem extends [Theorem 3.11, [12]] published by M. Jovanović, Z. Kadelburg and S. Radenović in 2010.

Theorem 4.3. Let $(X, d ; s)$ be a $b$-metric space with constant $s \geq 1$, and and let $S, T, I, J: X \rightarrow X$ be selfmappings satisfying the following conditions: 
(A 1) $S(X) \subset J(X)$ and $T(X) \subset I(X)$.

(A 2) The pairs $(S, I)$ and $(T, J)$ are weakly compatible.

(A 3)'One of the sets $S(X), T(X), I(X)$ or $J(X)$ is complete.

(A 4) There exists $\lambda \in\left(0, \frac{1}{s}\right)$ such that the inequality

$d(S x, T y) \leq \lambda \max \left\{d(I x, J y), d(I x, S x), d(J y, T y), \frac{d(I x, T y)}{2 s}, \frac{d(J y, S x)}{2 s}\right\}$,

holds for all $x, y \in X$.

Then $S, T, I$ and $J$ have a unique common fixed point (say) $z \in X$.

Furtheremore, we have $\{z\}=F i x\{S, I\}=F i x\{T, J\}$.

To obtain Theorem 4.3, on can use Theorem 3.2 and the function $f$ given by

$$
f\left(t_{1}, t_{2}, t_{3}, t_{4}, t_{5}\right):=\lambda \max \left\{t_{1}, t_{2}, t_{3}, \frac{t_{4}}{2 s}, \frac{t_{5}}{2 s}\right\}, \forall\left(t_{1}, t_{2}, t_{3}, t_{4}, t_{5}\right) \in[0,+\infty)^{5},
$$

with constant $\lambda \in\left[0, \frac{1}{s}\right)$. It is easy to verify that this function belongs to the set $\mathcal{F}_{s, 5}$. So we obtain the above theorem as a direct consequence of our second main result (i.e., Theorem 3.2).

The next theorem is a consequence of our main results.

Theorem 4.4. Let $(X, d ; s)$ be a complete b-metric space with constant $s \geq 1$, and and let the pairs $(S, I)$ and $(T, J)$ be b-compatible pairs of selfmaps of $X$ satisfying

$d(S x, T y) \leq \lambda \max \left\{d(I x, J y), d(S x, I x), d(T y, J y), \frac{d(S x, J y)+d(T y, I x)}{2 s}\right\}$,

for all $x, y \in X$, where $0 \leq \lambda<\frac{1}{s}$.

We suppose that

(i) $S(X) \subseteq J(X), T(X) \subseteq I(X)$;

(ii) the pairs $(S, I)$ and $(T, J)$ are weakly compatible and

(ii) $\overline{S(X)} \cap \overline{T(X)} \subset I(X) \cup J(X)$.

Then $I, J, S$ and $T$ have a unique common fixed point (say) $z \in X$.

Furtheremore, we have $\{z\}=F i x\{S, I\}=F i x\{T, J\}$.

We point out that Theorem 4.4 may be considered as a variant (for noncontinuous maps) of the result [Theorem 2.1., [11]] published by N. Hussain, Z. D. Mitrović, S. Radenović in 2018. 
If we set $T=I=J=S$ in Theorem 4.4, then we recapture Theorem 3.4 of the article [19].

Another consequence of our main result is the following.

Theorem 4.5. Let $(X, d ; s)$ be a complete b-metric space with constant $s \geq 1$, and and let the pairs $(S, I)$ and $(T, J)$ be b-compatible pairs of selfmaps of $X$ satisfying

$$
d(S x, T y) \leq a d(S x, I x)+b d(T y, J y)+c d(I x, J y)
$$

for all $x, y \in X$, where $a, b, c$ are non-negative real numbers satisfying $a+$ $s(b+c)<1$.

We suppose that

(i) $S(X) \subseteq J(X), T(X) \subseteq I(X)$;

(ii) the pairs $(S, I)$ and $(T, J)$ are weakly compatible and

(ii) $\overline{S(X)} \cap \overline{T(X)} \subset I(X) \cup J(X)$.

Then $I, J, S$ and $T$ have a unique common fixed point (say) $z \in X$.

Furtheremore, we have $\{z\}=F i x\{S, I\}=F i x\{T, J\}$.

This theorem is consequence of Theorem 3.1 with the function $f$ given by

$$
f\left(t_{1}, t_{2}, t_{3}, t_{4}, t_{5}\right):=c t_{1}+a t_{2}+b t_{3}, \forall\left(t_{1}, t_{2}, t_{3}, t_{4}, t_{5}\right) \in[0,+\infty)^{5},
$$

where $a, b, c$ are non-negative real numbers satisfying $a+s(b+c)<1$.

Indeed, it is easy to see that $f$ belongs to the class $\mathcal{F}_{s, 5}$. So, we may recapture this result as a consequence of the main theorem (Theorem 3.1) of this paper.

Let $I_{X}$ be the identity map of $X$. We observe that, if we set $T=S$ and $I=J=I_{X}$ in Theorem 4.5, then we recapture the main result (i.e Theorem 3.2) established in [17] by P. K. Mishra, S. Sachdeva and S. K. Banerjee.

Remark. It is possible to quote many other well known common fixed point results in b-metric spaces as consequences of the main results of this paper. We omit the details. 


\section{References}

[1] M. Akkouchi, "A common fixed point theorem connected to a result of V. Popa and H. K. Pathak", Georgian mathematical journal, vol. 13, no. 1, pp. 1-6, 2006, doi: 10.1515/ GMJ.2006.1

[2] M. Akkouchi, "Common fixed point theorems for two selfmappings of a b-metric space under an implicit relation", Hacettepe journal of mathematics and statistics, vol. 40, no. 6, pp. 805-810, 2011. [On line]. Available: https:// bit.ly/3cmWXg4

[3] M. Akkouchi, "A common fixed point theorem for expansive mappings under strict implicit conditions on b-metric spaces", Acta Universitatis Palackianae Olomucensis. Facultas Rerum Naturalium. Mathematica, vol. 50, no. 1, pp. 5-15, 2011. [On line]. Available: https:// bit.ly/3ckvvjf

[4] M. Akkouchi, "A common fixed point theorem for two pairs of weakly tangential maps in b-metric spaces", Journal of the International Mathematical Virtual Institute, vol. 9, pp. 189-204, 2019. [On line]. Available: https:// bit.ly/2FXthKn

[5] I. A. Bakhtin, "The contraction mapping principle in quasimetric spaces”, Functional analysis, vol. 30, pp. 26-37, 1989.

[6] V. Berinde, "Generalized contractions in quasimetric spaces," in Seminar on fixed point theory, vol. 3, Cuj-Napoca: "Babeg-Bolyai" University, Faculty of Mathematics and Computer Science, 1993, pp. 3-9. [On line]. Available: https:// bit.ly/2ZX4uNS

[7] S. Cobzaş, "B-metric spaces, fixed points and Lipschitz functions, Apr. 2018. arXiv:1802.02722v2

[8] S. Czerwik, "Contraction mappings in b-metric spaces", Acta mathematica et informatica Universitatis Ostraviensis, vol. 1, no. 1, pp. 5-11, 1993. [On line]. Available: https:// bit.ly/3hT7LUt

[9] S. Czerwik, "Nonlinear set-valued contraction mappings in B-metric spaces", Atti del seminario matematico e fisico dell'Universita di Modena e Reggio Emilia, vol. 46, no. 2, pp. 263-276, 1998.

[10] T. Dosenović, M. Pavlović, and S. Radenović, "Contractive conditions in b-metric spaces", Vojnotehnicki glasnik, vol. 65, no. 4, pp. 851-865, 2017, doi: 0.5937/vojtehg65-14817

[11] N. Hussain, Z. D. Mitrović, and S. Radenović, "A common fixed point theorem of Fisher in b-metric spaces", Revista de la Real Academia de Giencias Exactas, Físicas y Naturales. Serie A. Matemáticas, vol. 113, no. 2, pp.949-956, 2018, doi: 10.1007/s 13398-018-0524-x. 
[12] M. Jovanović, Z. Kadelburg, and S. Radenović, "Common fixed point results in metric-type spaces", Fixed point theory and applications, vol. 2010, no. 1, Art ID. 978121,2010, doi: 10.1155/2010/978121

[13] G. Jungck, "Compatible mappings and common fixed points", International journal of mathematics and mathematical sciences, vol. 9, no. 4, pp. 771-779, 1986, doi: 10.1155/S0161171286000935

[14] G. Jungck, "Common fixed points for noncontinuous nonself maps on nonmetric spaces", Far eastern journal of mathematics and science, vol. 4, no. 2, pp. 199-215, 1996.

[15] W. Kirk and N. Shahzad, Fixed point theory in distance spaces. Cham: Springer International Publishing, 2014, doi: 10.1007/978-3-31910927-5

[16] R. Miculescu and A. Mihail, "Caristi-Kirk type and Boyd and WongBrowder-Matkowski-Rus type fixed point results in b-metric spaces", Filomat, vol. 31, no. 14, pp. 4331-4340, 2017, doi: 10.2298/ FIL1714331M

[17] P. K. Mishra, S. Sachdeva, and S. K. Baner jee, "Some fixed point theorems in b-metric space", Turkish journal of analysis and number theory, vol. 2, no. 1, pp. 19-22, 2014, doi: 10.12691/t jant-2-1-5

[18] M. Paluszyński and K. Stempak, "On quasi-metric and metric spaces", Proceeding American Mathematical Society, vol. 137, no. 12, pp. 4307-4312, Dec. 2009, doi: 10.1090/S0002-9939-09-10058-8

[19] M. Sarwar and M.U. Rahman, "Fixed point theorems for Ciric's and generalized contractions in b-metric spaces", International journal of analysis and applications, vol. 7, no. 1, pp. 70-78, 2015. [On line]. Available: https:// bit.ly/3hQBmOp

[20] T. Suzuki, "Basic inequality on a b-metric space and its applications", Journal of inequalities and applications, vol. 2017, Art ID. 256, Oct. 2017, doi: 10.1186/s13660-017-1528-3 